\title{
Bortezomib-induced severe pulmonary complications in multiple myeloma: A case report and literature review
}

\author{
JINGBO LI* , SHUDA CHEN ${ }^{*}$, YINGHONG HU and JING CAI \\ Intensive Care Unit, The Second Affiliated Hospital, Zhejiang University College of Medicine, \\ Hangzhou, Zhejiang 310009, P.R. China
}

Received December 22, 2014; Accepted January 22, 2016

DOI: $10.3892 / \mathrm{ol} .2016 .4204$

\begin{abstract}
The proteasome inhibitor bortezomib is indicated for use in the treatment of multiple myeloma (MM) patients. The most common side effects are neurological and gastrointestinal, while severe pulmonary complications are rarely described. The present study reports the case of a 62-year-old man with immunoglobulin (Ig)G-type MM who was treated with bortezomib, thalidomide and dexamethasone. Subsequent to the administration of chemotherapy, the patient developed an acute respiratory distress syndrome. High-resolution computed tomography of the chest showed bilateral diffuse alveolar infiltrations and multiple subpleural lesions. A diagnosis of bortezomib-induced severe pulmonary complications was formed. Systemic corticosteroid therapy led to a rapid improvement in clinical conditions and radiological findings. In addition, the present study reviewed the characteristics, including medical history, clinical manifestations, treatment strategies and outcomes, of all $16 \mathrm{MM}$ patients with bortezomib-induced severe pulmonary complications reported previously in Pubmed. It was indicated that patients who were male, of $\mathrm{IgG}$ type, with a relapse status and a previous history of auto-PBSCT had a higher possibility of developing bortezomib-induced severe pulmonary complications. Additionally, a relatively low dose rather than a high dose of corticosteroids could obtain a better outcome.
\end{abstract}

Correspondence to: Dr Jingbo Li or Dr Jing Cai, Intensive Care Unit, The Second Affiliated Hospital, Zhejiang University College of Medicine, 88 Jiefang Road, Hangzhou, Zhejiang 310009, P.R. China E-mail: goldentongli@163.com

E-mail: jintongli@hotmail.com

*Contributed equally

Abbreviations: MM, multiple myeloma; HRCT, high-resolution computed tomography; CRP, C-reactive protein; PCT, procalcitonin; auto-PBSCT, autologous peripheral blood stem cell transplantation

Key words: bortezomib, multiple myeloma, pulmonary complications, corticosteroids

\section{Introduction}

Multiple myeloma (MM) is the second most common hematological cancer, and results from the accumulation of malignant plasma cells in the bone marrow (1). MM is responsible for $\sim 1 \%$ of all cancers and $10 \%$ of hematological cancers $(1,2)$. The median age at diagnosis of MM is 70 years (3). MM is associated with a number of complications, which include: Hematological complications, such as anemia, bone marrow failure and bleeding disorders; bone complications, such as pathological fractures and lytic bone lesions; renal insufficiencies; compromised immune function; and neurological complications, such as spinal cord and nerve root compression and cranial nerve compression, which are often associated with base of skull lesions (1). The median survival of patients with MM was $<1$ year prior to the introduction of alkylating agents (4). The introduction of novel agents, including bortezomib, thalidomide and lenalidomide, for the treatment of MM patients has significantly improved clinical outcomes $(3,5)$.

Bortezomib is a proteasome inhibitor that exhibits significant anti-myeloma activity. The majority of adverse effects associated with bortezomib are mild or moderate and are generally manageable, including gastrointestinal symptoms, peripheral neuropathy and thrombocytopenia (6). Severe pulmonary complications have rarely been reported. The present study reports the case of a patient in whom life-threatening pulmonary adverse effects developed after receiving bortezomib combined with thalidomide and dexamethasone for newly diagnosed MM.

\section{Case report}

A 62-year-old man was referred to The Second Affiliated Hospital, Zhejiang University College of Medicine (Hangzhou, Zhejiang, China) due to dizziness and weakness that had been apparent for 3 months, since August 2013. The patient had a 9-year history of coronary heart disease, for which the standard medications (aspirin, $100 \mathrm{mg} /$ day; atorvastatin, $20 \mathrm{mg} / \mathrm{day}$; metoprolol, $25 \mathrm{mg} /$ day; all for 9 years) were administered, and a $>30$-year history of smoking, which the patient quitted after being diagnosed with coronary heart disease. There was no history of respiratory diseases. At the cardiological clinic in The Second Affiliated Hospital, Zhejiang University 
College of Medicine, the patient was found to have moderate anemia. Furthermore, serological tests and bone marrow studies confirmed the pathologic diagnosis of MM [IgG type; Durie-Salmon, stage III (7)]. The patient was consequently admitted for chemotherapy.

Treatment with bortezomib $\left(1 \mathrm{mg} / \mathrm{m}^{2}\right.$ on days $1,4,8$ and 11), thalidomide (100 mg/day) and dexamethasone $(20 \mathrm{mg}$ on days $1,2,4,5,8,9,11$ and 12) was employed in a 21-day cycle. The initial chest high-resolution computed tomography (HRCT) scan was normal. The first cycle was effective and uneventful. However, on the eve of the second cycle, the patient developed a moderate fever $\left(38.5^{\circ} \mathrm{C}\right)$ and a mild dry cough. Despite the fact that the leukocyte count and lung auscultation were normal, moxifloxacin (400 mg/day) was prescribed to prevent probable pneumonia. The fifth and sixth agents of bortezomib were administered on schedule. After the sixth agent, the patient's temperature ascended to $39.1^{\circ} \mathrm{C}$, and auscultation revealed crackles in the left lung. The chest HRCT scan demonstrated a newly developed consolidation in the upper lobe and infiltration in the lower lobe of the left lung (Fig. 1). Pneumonia was initially considered due to the immunosuppressive status of the patient, and intravenous broad-spectrum antibiotics (including $500 \mathrm{mg}$ imipenem/cilastatin every $8 \mathrm{~h}$, $1,000 \mathrm{mg}$ vancomycin every $12 \mathrm{~h}$ and $200 \mathrm{mg}$ voriconazole every $12 \mathrm{~h}$ ) were administered.

However, the patient's clinical condition worsened following the treatment. The next day, asthma-like symptoms and mild dyspnea were noted, and the patient began to expectorate bloody sputum. The high fever remained (maximum temperature, $\left.39^{\circ} \mathrm{C}\right)$. Acute respiratory distress $\left(\mathrm{SpO}_{2}, 85 \%\right)$ on room air developed 3 days later. The chest HRCT scan showed bilateral diffuse alveolar infiltrations, multiple subpleural lesions and slight pleural effusion (Fig. 2). The chemotherapy was discontinued due to the patient's poor clinical condition. Infection by virus and/or Pneumocystis jiroveci could not be definitively excluded; therefore, ganciclovir (300 mg every $12 \mathrm{~h}$ for 3 days) and trimethoprim/sulfamethoxazole (960 mg every $12 \mathrm{~h}$ for 3 days) were added into the therapy. Intravenous immunoglobulin (10 g/day for 6 days; then $5 \mathrm{~g}$ /day for 3 days) was also administered to strengthen the patient's immunity. The patient was then transferred into the Intensive Care Unit.

Despite receiving a large number of powerful antibiotics, the patient's clinical status did not improve. The patient retained an $\mathrm{SpO}_{2}$ level of $92 \%$ when masked with a reservoir bag, and a flow rate of $15 \mathrm{l} / \mathrm{min}$ oxygen. Serological tests, including repeated $\mathrm{C}$-reactive protein (CRP) and procalcitonin (PCT) analysis, were normal. The bacteriological and mycological cultures of the sputum and blood samples were negative. No invasive investigations, such as bronchoalveolar lavage or transbronchial biopsy, were performed due to thrombocytopenia and the critical condition of the patient. A suspected diagnosis of a bortezomib-induced severe pulmonary complication was formed, and $120 \mathrm{mg} /$ day methylprednisolone was instigated. The respiratory failure was relieved 5 days later and a normal temperature was recorded. The dose of methylprednisolone was tapered $(120 \mathrm{mg} /$ day for 2 days; $80 \mathrm{mg} /$ day for 2 days; $40 \mathrm{mg} /$ day for 2 days; $30 \mathrm{mg} /$ day for 2 days; $20 \mathrm{mg} /$ day for 2 days; then discontinued), the patient's clinical condition improved gradually and the results on chest HRCT scan improved synchronously
(Fig. 3). Another 18 days later, the patient was discharged without any respiratory symptoms.

Written informed consent was obtained from the patient for publication of this case report and any accompanying images.

\section{Discussion}

The first study of MM patients who developed life-threatening pulmonary complications after receiving bortezomib therapy reported the cases of 4 Japanese patients (6). Since this time, 16 cases have been described as MM with bortezomib-induced pulmonary complications $(6,8-15)$. The patient in the present study also developed severe pulmonary complications during bortezomib treatment. The differential diagnosis included infectious pneumonia and drug-induced pulmonary toxicity. The clinical course in this case was similar to those previously reported cases. The patient developed symptoms that included a fever, a cough, bloody sputum, gradual dyspnea, and ultimately, respiratory failure. The chest HRCT scan showed diffuse infiltration and consolidation of the bilateral lungs, accompanied with slight pleural effusion. The inflammatory markers, CRP and PCT, were normal. All the cultures of the blood and sputum were negative. Powerful broad-spectrum antibiotics were inefficacious. Therefore, the patient was diagnosed with bortezomib-induced severe pulmonary complications. Although no histopathological evaluation was obtained to prove the diagnosis, a good response to corticosteroids favored the diagnosis, as observed in previous reports $(9,12,15)$.

The clinical characteristics of all 16 reported patients are listed in Table I. The cases consisted of 10 male patients $(62.5 \%)$ and 6 female patients $(37.5 \%)$. The mean age was 62.3 years (range, $47-74$ years). Only 3 patients $(18.8 \%$ ) had a previous history of respiratory diseases [including invasive pulmonary aspergillosis (6), pulmonary embolism (6) and chronic obstructive pulmonary disease (9)], while $11(68.8 \%)$ had not, and 2 had an unknown history. In total, 9 patients $(56.3 \%)$ were of IgG type, 2 (12.5\%) of IgD type, $1(6.3 \%)$ of $\mathrm{IgA}$ type, $1(6.3 \%)$ of IgC type and $3(18.8 \%)$ of unknown type. The cases consisted of 10 relapsed patients $(62.5 \%)$, 4 newly diagnosed patients $(25 \%)$ and 2 refractory patients $(12.5 \%)$. A total of 6 patients $(37.5 \%)$ had received autologous peripheral blood stem cell transplantation (auto-PBSCT) prior to treatment with bortezomib, 1 (6.3\%) had received an unrelated bone marrow transplantation, 4 (25\%) had no transplantation history and $5(31.3 \%)$ had an unknown transplantation history. Therefore, the male gender, IgG type, relapse state and previous history of auto-PBSCT may be risk factors for bortezomib-induced severe pulmonary complications in MM.

In the 16 cases, the mean number of bortezomib injections administered prior to severe pulmonary complications appearing was 6.9 (range, 1-32), and the mean time from the first administration of bortezomib to the appearance of severe pulmonary complications was 31.3 days (range, 1-161 days) (Table II).

Themostcommon clinical symptoms of bortezomib-induced severe pulmonary complications were dyspnea $(13 / 16 ; 81.3 \%)$, respiratory failure $(13 / 16 ; 81.3 \%)$, a fever $(10 / 16 ; 62.5 \%)$ and a cough $(5 / 16 ; 31.3 \%)$. The most common radiological findings 


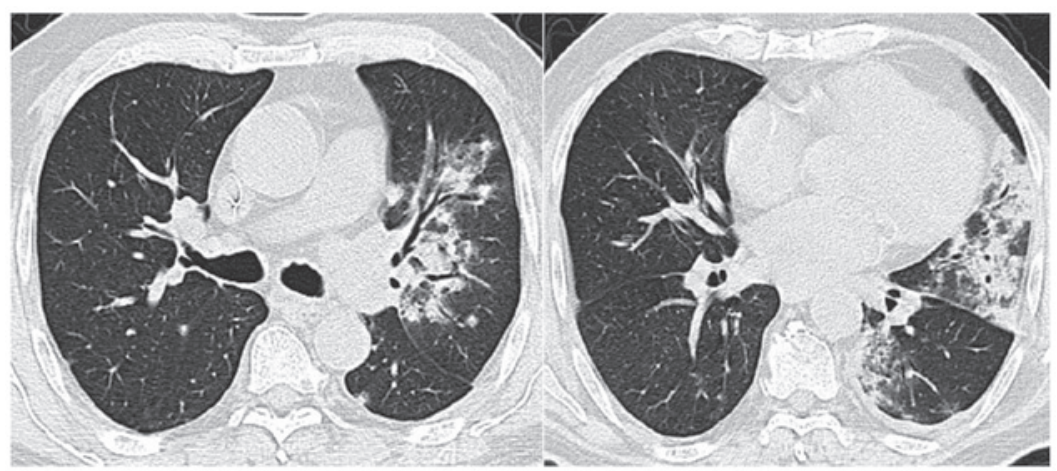

Figure 1. Chest high-resolution computed tomography scan on the day of the sixth dose of bortezomib showing newly developed consolidation and infiltration in the left lung.

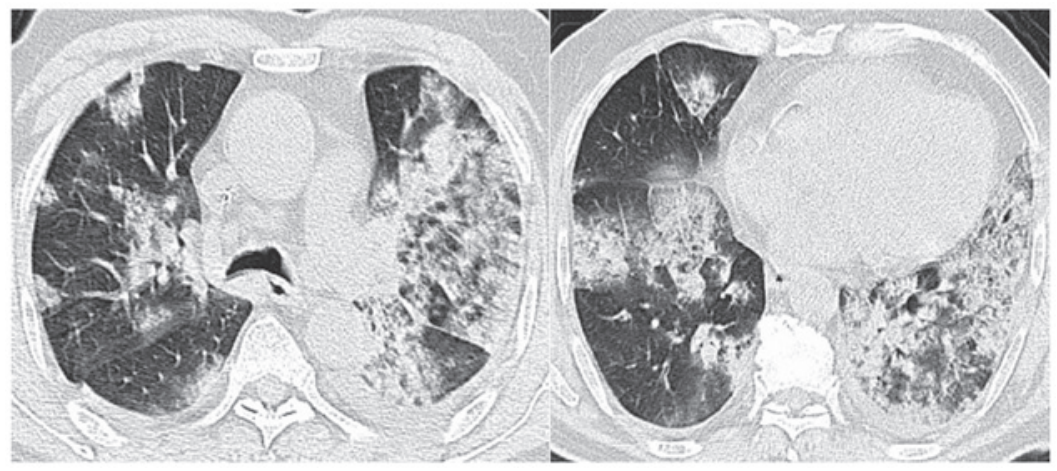

Figure 2. Chest high-resolution computed tomography scan 3 days after the sixth dose of bortezomib showing rapidly deteriorating lung injury with bilateral diffuse alveolar infiltration and multiple subpleural lesions.

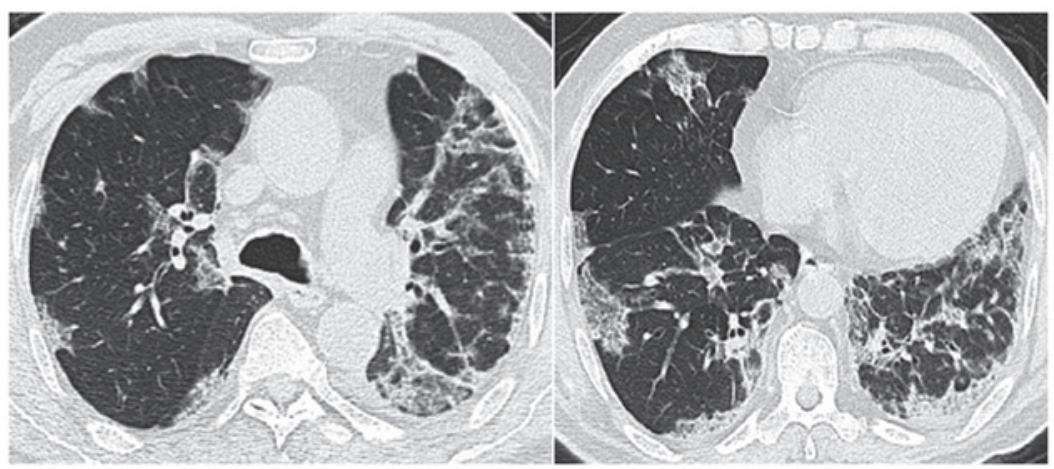

Figure 3. Chest high-resolution computed tomography scan 12 days after starting corticosteroid treatment showing marked improvement of the lung infiltration.

were pulmonary infiltration $(9 / 16 ; 56.3 \%)$, pleural effusion $(6 / 16 ; 37.5 \%)$, pulmonary edema $(4 / 16 ; 25 \%)$ and ground-glass opacities $(4 / 16 ; 25 \%)$ (Table II).

Due to the poor clinical condition of the patients, only $4(25 \%)$ achieved a pathological diagnosis [organizing diffuse alveolar damage (6), fibrosis with admixed chronic inflammatory cells (8), diffuse interstitial fibrosis (11) and bronchiolitis obliterans with organizing fibroblastic polyps in alveoli (13), respectively] that totally accounted for the changes of the pulmonary interstitium. The inner mechanism of bortezomib-induced severe pulmonary complications remains unclear. However, the following pathogenetics have been involved in the development of lung injury: i) Activation nuclear factor- $\mathrm{\kappa B}$-related proinflammatory factors, including tumor necrosis factor- $\alpha$, interleukin (IL)-6, IL-1 $\beta$ and IL-11; and ii) accumulation of bortezomib and/or its metabolites, altering multiple signaling pathways $(6,10,13)$.

All patients received corticosteroid therapy following the occurrence of severe pulmonary complications, however, while half of these patients experienced improvement, the other half succumbed. Among the 7 patients in whom corticosteroid types or doses were not precisely mentioned, 6 patients (85.7\%) succumbed and 1 patient (14.3\%) improved (Table II).

The first hypothesis of proinflammatory factors would explain the good response to corticosteroid therapy in certain patients $(9,13)$; however, other patients developed severe pulmonary complications even when receiving dexamethasone in combination with bortezomib. In all 16 
Table I. Characteristics of $16 \mathrm{MM}$ patients with bortezomib-induced severe pulmonary complications.

\begin{tabular}{|c|c|c|c|c|c|c|c|}
\hline First author, year & $\begin{array}{l}\text { Age, } \\
\text { years }\end{array}$ & Gender & MM type & $\begin{array}{l}\text { Previous history } \\
\text { of lung injury }\end{array}$ & $\begin{array}{l}\text { Status of MM prior to } \\
\text { bortezomib therapy }\end{array}$ & $\begin{array}{c}\text { Prior } \\
\text { transplantation }\end{array}$ & Ref. \\
\hline \multirow[t]{4}{*}{$\begin{array}{l}\text { Miyakoshi et al, } \\
2006\end{array}$} & 47 & $\mathrm{~F}$ & $\operatorname{IgG}$ & $\begin{array}{c}\text { Invasive pulmonary } \\
\text { aspergillosis }\end{array}$ & Relapse & Auto-PBSCT & (6) \\
\hline & 59 & $\mathrm{~F}$ & $\operatorname{IgA}$ & Pulmonary embolism & Relapse & Auto-PBSCT & \\
\hline & 48 & $\mathrm{~F}$ & $\operatorname{IgD}$ & No & Relapse & $\begin{array}{l}\text { Unrelated bone } \\
\text { marrow transplantation }\end{array}$ & \\
\hline & 53 & $\mathrm{~F}$ & Unknown & No & Relapse & Auto-PBSCT & \\
\hline Boyer et al, 2006 & 66 & M & Unknown & No & Relapse & No & (8) \\
\hline $\begin{array}{l}\text { Zappasodi et al, } \\
2007\end{array}$ & 66 & M & Unknown & COPD & Refractory & Unknown & (9) \\
\hline \multirow[t]{5}{*}{ Dun et al, 2010} & 72 & M & $\operatorname{IgG}$ & No & Relapse & Unknown & $(10)$ \\
\hline & 51 & M & $\operatorname{IgD}$ & No & Refractory & Unknown & \\
\hline & 72 & $\mathrm{~F}$ & $\operatorname{IgG}$ & No & New & No & \\
\hline & 68 & $\mathrm{~F}$ & $\operatorname{IgG}$ & No & Relapse & Unknown & \\
\hline & 72 & M & $\operatorname{IgG}$ & No & Relapse & Unknown & \\
\hline Kang et al, 2010 & 67 & M & $\operatorname{IgG}$ & Unknown & New & No & (11) \\
\hline $\begin{array}{l}\text { Yamaguchi et al, } \\
2012\end{array}$ & 64 & M & $\operatorname{IgG}$ & No & Relapse & Auto-PBSCT & (12) \\
\hline Vandeix et al, 2012 & 74 & M & $\operatorname{IgC}$ & No & New & No & (13) \\
\hline Chew et al, 2007 & 66 & M & $\operatorname{IgG}$ & Unknown & New & Auto-PBSCT & (14) \\
\hline Pitini et al, 2007 & 51 & M & $\operatorname{IgG}$ & No & Relapse & Auto-PBSCT & (15) \\
\hline
\end{tabular}

Ig, immunoglobulin; COPD, chronic obstructive pulmonary disease; auto-PBSCT, autologous peripheral blood stem cell transplantation; M, male; F, female; MM, multiple myeloma.

reported patients, $10(62.5 \%)$ were administered corticosteroids concurrently with bortezomib, while 5 (31.3\%) were not and in 1 case this was unknown. Among the 10 cases that received bortezomib combined with corticosteroids, $4(40 \%)$ showed improvement and $6(60 \%)$ succumbed when retreated with corticosteroids. However, among the 5 cases that received bortezomib without corticosteroids, $3(60 \%)$ showed improvement and $2(40 \%)$ succumbed. Despite the fact that retreatment with corticosteroids remained efficacious, it appeared that outcomes of patients who received corticosteroids combined with bortezomib were worse than those treated with bortezomib only. This would suggest the existence of another unknown complex pathogenesis. Further investigations are required to elucidate the underlying reasons.

In a number of the early published studies $(6,9,15)$, the patients mainly received high-dose corticosteroids (500-1,000 mg/day methylprednisolone), but the outcomes were not totally satisfactory. Among the 6 patients who received high-dose corticosteroids, 4 (66.7\%) showed improvement and $2(33.3 \%)$ succumbed. However, low-dose corticosteroids (1 mg/kg/day methylprednisolone/prednisolone) showed a good response in several cases $(8,12,13)$. All 3 of the patients who received low-dose steroids showed improvement. In the present case, success was also achieved with $120 \mathrm{mg}$ methylprednisolone initially.

In two particular cases reported by Miyakoshi et al (6) and Chew et al (14), the patients experienced two episodes of drug-induced pulmonary adverse effects in their whole course of bortezomib therapy. The first pulmonary complications improved spontaneously or through treatment with corticosteroid. However, severe pulmonary complications developed again and the patients ultimately succumbed when retreated with bortezomib. As a result, we hypothesize that retreating patients with bortezomib after a 'sentinel' episode of lung injury, a history of pulmonary complications during the previous treatment with bortezomib, could be fatal and should be avoided.

In conclusion, clinicians should be aware of potential severe pulmonary complications in patients receiving bortezomib, even when combined with dexamethasone, and particularly in patients who are male, of IgG type, with a relapse status and a history of auto-PBSCT. In the literature review, it was noted that when severe pulmonary complications were present, corticosteroids remained the only effective drug. It appears that the outcomes of the patients who received corticosteroids combined with bortezomib were worse than those for patients treated with bortezomib only. Considering the adverse effects associated with high-dose corticosteroids, a relatively low dose could be more suitable. At the same time, bortezomib should be discontinued immediately. Retreating patients with bortezomib after a 'sentinel' episode of lung injury could be fatal and should be avoided.

\section{Acknowledgements}

The authors would like to thank Dr Lili Yang and Dr Rongwang Yang (The Children's Hospital, Zhejiang University College of Medicine) for copy-editing the original manuscript. 


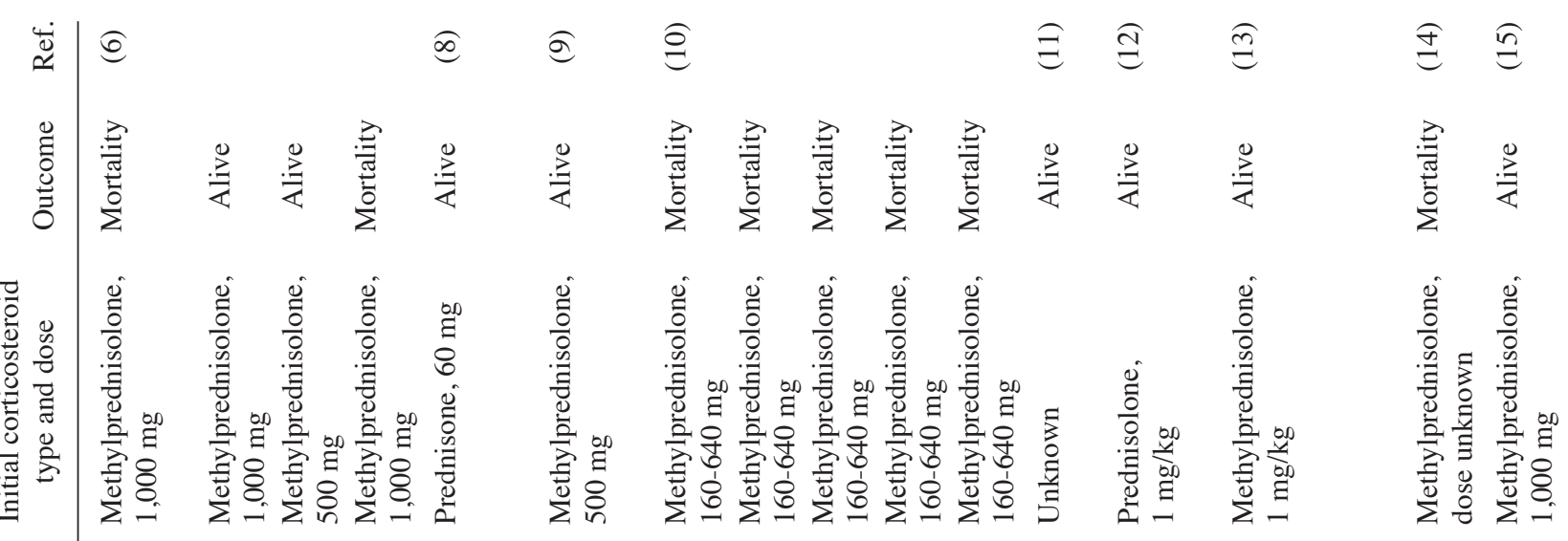

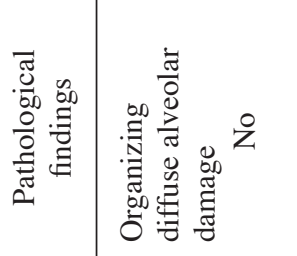

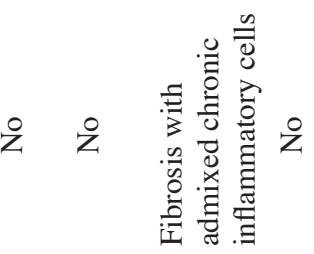

ż ஜ

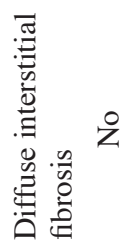

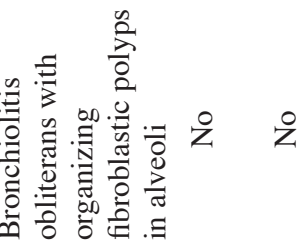

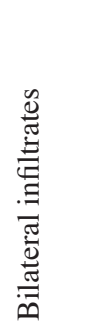

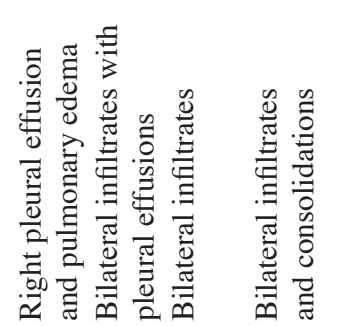

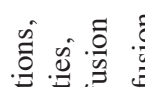

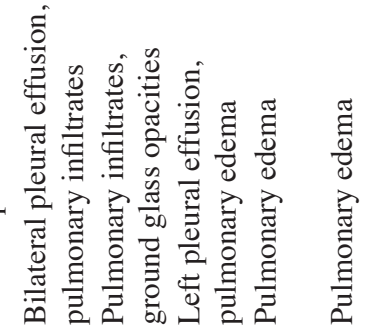
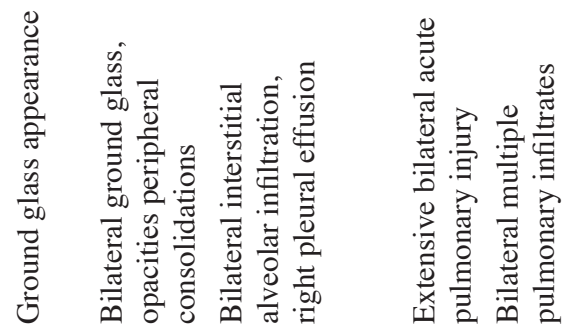

竞竞<smiles>C1C2CC1C2</smiles>

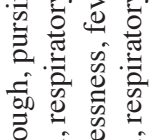

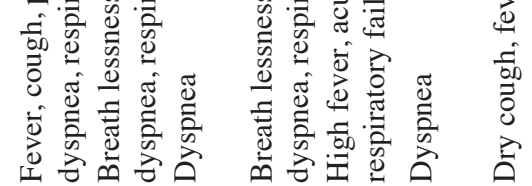

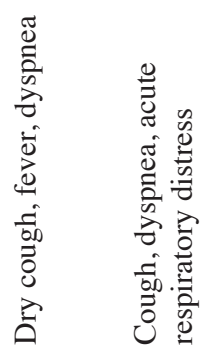

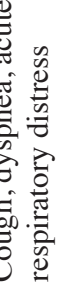

总玤

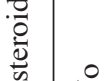
$\stackrel{\circ}{z}$

总

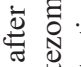

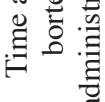

危

$\begin{array}{ll} & \\ 0 & 0 \\ 0 & 0 \\ 0 & 0 \\ 0 & 0 \\ 0\end{array}$

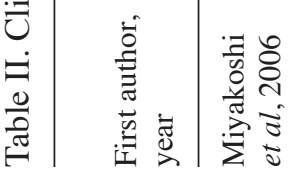

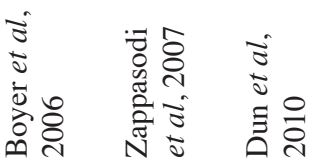

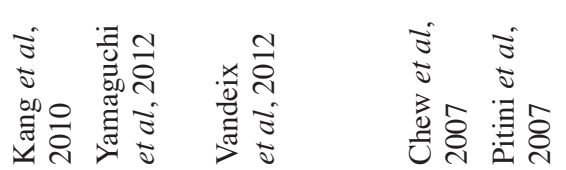




\section{References}

1. Smith L, McCourt O, Henrich M, Paton B, Yong K, Wardle J and Fisher A: Multiple myeloma and physical activity: A scoping review. BMJ Open 5: e009576, 2015.

2. Kyle RA and Rajkumar SV: Multiple myeloma. Blood 111: 2962-2972, 2008.

3. Gozzetti A, Candi V, Papini G and Bocchia M: Therapeutic advancements in multiple myeloma. Front Oncol 4: 241, 2014.

4. Kumar SK, Rajkumar SV, Dispenzieri A, Lacy MQ, Hayman SR, Buadi FK, Zeldenrust SR, Dingli D, Russell SJ, Lust JA, et al: Improved survival in multiple myeloma and the impact of novel therapies. Blood 111: 2516-2520, 2008.

5. Fulciniti M, Munshi NC and Martinez-Lopez J: Deep response in multiple myeloma: A critical review. Biomed Res Int 2015: 832049, 2015.

6. Miyakoshi S, Kami M, Yuji K, Matsumura T, Takatoku M, Sasaki M, Narimatsu H, Fujii T, Kawabata M, Taniguchi S, et al: Severe pulmonary complications in Japanese patients after bortezomib treatment for refractory multiple myeloma. Blood 107: 3492-3494, 2006.

7. Hari PN, Zhang MJ, Roy V, Pérez WS, Bashey A, To LB, Elfenbein G, Freytes CO, Gale RP, Gibson J, et al: Is the International Staging System superior to the Durie-Salmon staging system? A comparison in multiple myeloma patients undergoing autologous transplant. Leukemia 23: 1528-1534, 2009.
8. Boyer JE, Batra RB, Ascensao JL and Schechter GP: Severe pulmonary complication after bortezomib treatment for multiple myeloma. Blood 108: 1113, 2006.

9. Zappasodi P, Dore R, Castagnola C, Astori C, Varettoni M, Mangiacavalli S, Lazzarino M and Corso A: Rapid response to high-dose steroids of severe bortezomib-related pulmonary complication in multiple myeloma. J Clin Oncol 25: 3380-3381, 2007.

10. Dun XY, Yuan ZG, Fu WJ, Zhang CY and Hou J: Severe pulmonary complications after bortezomib treatment in multiple myeloma. Hematol Oncol 28: 49-52, 2010.

11. Kang W, Kim JS, Cho SH, Kim SK, Chang J and Park MS: Nonspecific interstitial pneumonitis after bortezomib and thalidomide treatment in a multiple myeloma patient. Yonsei Med J 51: 448-450, 2010.

12. Yamaguchi T, Sasaki M and Itoh K: Bortezomib-induced pneumonitis during bortezomib retreatment in multiple myeloma. Jpn J Clin Oncol 42: 637-639, 2012.

13. Vandeix E, Favard F, Pichon N, Delage-Corre M, Melloni B and Clavel M: Bortezomib-induced bronchiolitis obliterans organizing pneumonia. Case Rep Pulmonol 2012: 430141, 2012.

14. Chew E, Filsher R and Wei A: Development of fatal bortezomib induced acute lung injury despite concurrent therapy with high-dose dexamethasone. Leuk Lymphoma 48: 212-213, 2007.

15. Pitini V, Arrigo C, Altavilla G and Naro C: Severe pulmonary complications after bortezomib treatment for multiple myeloma: An unrecognized pulmonary vasculitis? Leuk Res 31: 1027-1028, 2007. 